\title{
Universal Scaling Laws for Dense Particle Suspensions in Turbulent Wall-Bounded Flows
}

\author{
Pedro Costa, ${ }^{1, *}$ Francesco Picano, ${ }^{2}$ Luca Brandt, ${ }^{3}$ and Wim-Paul Breugem ${ }^{1}$ \\ ${ }^{1}$ Laboratory for Aero and Hydrodynamics, Delft University of Technology, Leeghwaterstraat 21, NL-2628 CA Delft, Netherlands \\ ${ }^{2}$ Department of Industrial Engineering, University of Padova, Via Venezia 1, 35131 Padua, Italy \\ ${ }^{3}$ SeRC (Swedish e-Science Research Centre) and Linné FLOW Centre, KTH Mechanics, SE-100 44 Stockholm, Sweden
}

(Received 6 January 2016; published 20 September 2016)

\begin{abstract}
The macroscopic behavior of dense suspensions of neutrally buoyant spheres in turbulent plane channel flow is examined. We show that particles larger than the smallest turbulence scales cause the suspension to deviate from the continuum limit in which its dynamics is well described by an effective suspension viscosity. This deviation is caused by the formation of a particle layer close to the wall with significant slip velocity. By assuming two distinct transport mechanisms in the near-wall layer and the turbulence in the bulk, we define an effective wall location such that the flow in the bulk can still be accurately described by an effective suspension viscosity. We thus propose scaling laws for the mean velocity profile of the suspension flow, together with a master equation able to predict the increase in drag as a function of the particle size and volume fraction.
\end{abstract}

DOI: 10.1103/PhysRevLett.117.134501

Turbulent, wall-bounded suspensions appear widely in environmental and industrial contexts. These suspensions are often dense; i.e. the volume fraction is sufficiently high that particle-particle and particle-fluid interactions strongly influence the macroscopic flow dynamics. In many cases, the suspended particles have a finite size-comparable to or larger than the smallest scales in the flow, and particle inertia plays an important role [1].

The flow of suspensions under laminar conditions has been thoroughly studied since Einstein [2] analytically derived an expression for the effective viscosity of a suspension of rigid spheres in the dilute and viscous limit: $\nu^{e} / \nu=1+(5 / 2) \Phi$, where $\nu$ is the kinematic viscosity of the suspending fluid, and $\Phi$ the bulk solid volume fraction. In dense cases, the rheology of laminar suspensions is usually characterized by semiempirical formulas for the effective viscosity $[3,4]$.

When the Reynolds number (which quantifies the importance of fluid inertial to viscous effects) is sufficiently high, the flow becomes turbulent, exhibiting chaotic and multiscale dynamics. Wall-bounded turbulent flows are characterized by at least one inhomogeneous direction and by the constraint of vanishing velocity at the wall, which makes their analysis even more complicated. For simplicity, we consider the canonical case of a pressure-driven turbulent plane-channel flow laden with neutrally buoyant particles, defined by the bulk Reynolds number $\operatorname{Re}_{b}=U_{b} 2 h / \nu$, where $U_{b}$ is the bulk velocity (i.e. averaged over the entire domain) and $h$ the half channel height. In the single-phase limit, the most well-known results from classical turbulence theory are the scaling laws for the mean velocity and the associated drag, or pressure loss. This is obtained by dividing the flow into two regions: the inner layer, close to the wall, $y \ll h$, with relevant velocity and length scales $u_{\tau}$ and $\delta_{v}$, and the outer layer, away from the wall, $y \gg \delta_{v}$, governed by $u_{\tau}$ and $h$; here $u_{\tau}=\sqrt{\tau_{w} / \rho}$ is the friction velocity, $\tau_{w}$ the wall shear stress, $\delta_{v}=\nu / u_{\tau}$ the viscous wall unit, and $\rho$ the fluid mass density.

At high-enough friction Reynolds number, $\operatorname{Re}_{\tau}=h / \delta_{v}=u_{\tau} h / \nu \gtrsim 100$, corresponding to $\operatorname{Re}_{b} \gtrsim$ 3000 [5], an overlap region exists, $\delta_{v} \ll y \ll h$. Here a logarithmic law can be derived for the inner-scaled mean velocity profile, $u / u_{\tau}=(1 / \kappa) \ln \left(y / \delta_{v}\right)+B$, and for the outer-scaled defect law $\left(U_{c}-u\right) / u_{\tau}=$ $-(1 / \kappa) \ln (y / h)+B_{d}$, with $U_{c}$ the centerline velocity, $\kappa \approx 0.41$ the so-called von Kármán constant, $B \approx 5.2$ and $B_{d} \approx 0.2$. These simple scaling laws, derived in 1930 [6], have been confirmed by many numerical and experimental studies (see e.g. [7] for a review). Their importance is unquestionable to predict the overall drag [8] and to use as a basis for many near-wall closure models currently used in computational fluid dynamics [9].

At the very high Reynolds numbers typically encountered in practice, the suspended particles are larger than the smallest turbulent scales $\left(\sim \delta_{v}\right)$ and the single-phase approach fails to reproduce the behavior of turbulent channel flows of dense suspensions even when accounting for an effective suspension viscosity [10-12].

In this Letter we propose scaling laws for turbulent wallbounded suspension flows. These are characterized by three parameters: the bulk Reynolds number $\operatorname{Re}_{b}$, the bulk solid volume fraction $\Phi$, and the particle diameter $D_{p} / h$. These laws are capable of predicting the mean velocity and drag from dilute to dense cases, from large to relatively small particles and for a wide range of Reynolds numbers.

We use data from interface-resolved direct numerical simulations (DNS). The DNS solve the Navier-Stokes equations for an incompressible Newtonian fluid in a plane 
TABLE I. Physical and computational parameters of the DNS database (consisting of 20 simulations). $N_{p}$ denotes the number of particles and $\delta_{v}^{s p h}\left(\gtrsim \delta_{v}\right)$ the viscous wall unit for the corresponding single-phase flow at the same $\operatorname{Re}_{b}$.

\begin{tabular}{lcrrrr}
\hline \hline Case & $h / D_{p}$ & $D_{p} / \delta_{v}^{s p h}$ & $\Phi(\%)$ & $\operatorname{Re}_{b}$ & \multicolumn{1}{c}{$N_{p}$} \\
\hline D10 & 36 & 9.7 & 20 & 12000 & 640000 \\
D20 & 18 & 19.4 & 20 & 12000 & 80000 \\
D10_2 & 36 & 9.7 & 5 & 12000 & 160000 \\
FP [11] & 9 & 19.9 & $0-20$ & 5600 & $0-10000$ \\
IL [18] & 5 & $20.7,32.4$ & $0-30$ & 3000,5000 & $0-2580$ \\
\hline \hline
\end{tabular}

channel with periodic boundary conditions in the streamwise $(x)$ and spanwise $(z)$ directions over lengths of $6 h$ and $3 h$, respectively, and no-slip and no-penetration at the bottom $(y=0)$ and top $(y=2 h)$ walls. The flow solver is extended with an immersed boundary method to force the fluid velocity to the local particle velocity at the particle surface [13]. Lubrication closures are used for short-range particle-particle and particle-wall interactions when intersurface distances are smaller than a grid cell and a softsphere collision model for solid-solid contacts $[14,15]$. The method has been tested and validated against several benchmark cases [15-17]. The flow is resolved on a uniform Cartesian grid with size $\Delta=D_{p} / 16$. The computational parameters are presented in Table I where we also report the cases from $[11,18]$ used here for comparison. The data are complemented with an unladen single-phase reference (SPR) case at the same $\operatorname{Re}_{b}=12000$ and a continuum limit reference (CLR), i.e. the single-phase flow of a fluid with the effective viscosity $\nu^{e}$ of a suspension with volume fraction $\Phi=0.2$, corresponding to $\operatorname{Re}_{b}^{e}=$ $\operatorname{Re}_{b} \nu / \nu^{e} \approx 6400$ in our case.

Figure 1 shows the mean velocity profile for $D_{p} / \delta_{v} \approx 10$ (D10) and 20 (D20), compared to the continuum limit reference. The comparison between the single-phase and the two-phase flows requires a proper definition of the viscous wall unit in terms of $\nu^{e}$, here $\delta_{v}^{e}=\nu^{e} / u_{\tau}$. Despite the improvement with respect to the use of the classical definition of $\delta_{v}=\nu / u_{\tau}$ (see the inset of Fig. 1), the figure reveals that the particle-laden flows show a clear deviation from the classical logarithmic law. The differences with the continuum limit are for larger particles, and so is the measured increase in drag. The abrupt change of the slope of the profile at a wall-normal distance of $y \sim D_{p}$ suggests that the deviation from the continuum limit is caused by a change in the near-wall dynamics. Studies of laminar wallbounded flows laden with neutrally buoyant spheres report a structured arrangement of particles near the wall $[16,19,20]$. This layering is attributed to the planar symmetry imposed by the wall and to stabilizing particleparticle and particle-wall interactions. Though more pronounced under laminar conditions, this phenomenon is also present in turbulent suspensions [11,18]. Figure 2(a) presents the mean local number density $n$, normalized with

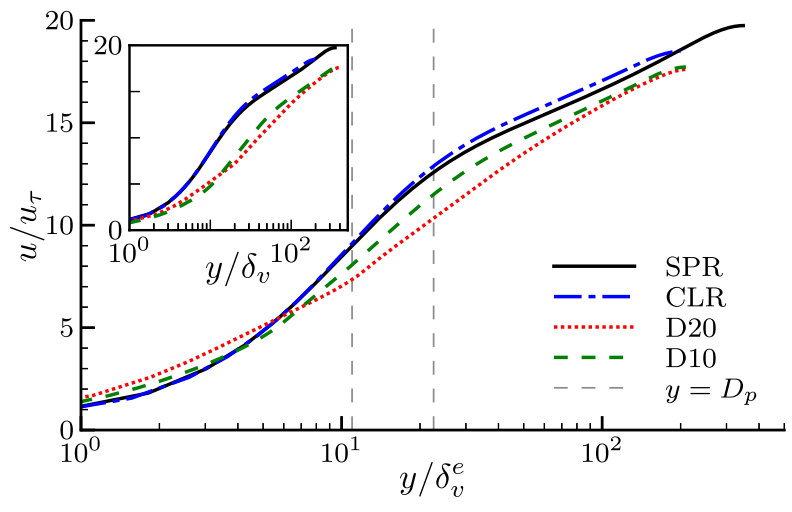

FIG. 1. Mean streamwise flow velocity, $u / u_{\tau}$, versus the wallnormal distance in inner scaling $y / \delta_{v}^{e}$. Vertical dashed lines depict a wall-normal distance of 1 particle diameter $\left(y=D_{p}\right)$ for cases D10 (closest to $y=0$ ) and D20; see Table I. Maximum statistical error within $95 \%$ confidence interval is $\pm 0.9 \%$. The inset shows the same velocity profile but with the wall-normal distance scaled with $\delta_{v}$.

the corresponding bulk value $N$, for cases D10 and D20 (see Table I). The particle layer is evident from the local minimum at a distance of one particle diameter from the wall, as shown in the inset where the horizontal axis is scaled with $D_{p}$.

The apparent mean particle-to-fluid slip velocity is highest close to the wall and becomes negligible at wallnormal distances $y \gtrsim D_{p}$; see Fig. 2(b) where we report the wall-normal profiles of the mean particle and fluid velocity for two of the cases considered. Away from the wall, the complex interaction between the turbulent fluid motion and the particles still result in approximately the same average value of streamwise velocity, as if the two phases behave as a continuum. The layer of particles near the wall shows an almost constant slip with respect to the fluid. This large slip indicates that continuum models based on an effective viscosity are bound to fail.

The inset of Fig. 2(b) reports the fluid velocity divided by the particle-to-fluid slip velocity at the wall, $u_{p w}$, versus the wall-normal distance in units of particle radius $R_{p}$. For the same volume fraction of $20 \%$, results from different numerical simulations with different Reynolds numbers and particle sizes collapse for wall-normal distances smaller than a particle radius. It appears that, in dense suspensions, a particle-wall layer exists that prevents a direct interaction between the turbulent suspension flow in the core and the solid wall underneath the particle-wall layer. This serves as a starting point for the scaling arguments presented hereafter.

The former considerations motivate a modeling approach based on the separation between the dynamics of the particle-wall layer and of the turbulent flow region. We will denote the latter as the homogeneous suspension region (HSR), meaning a well-mixed suspension. Let us therefore define the thickness of the particle-wall layer by 

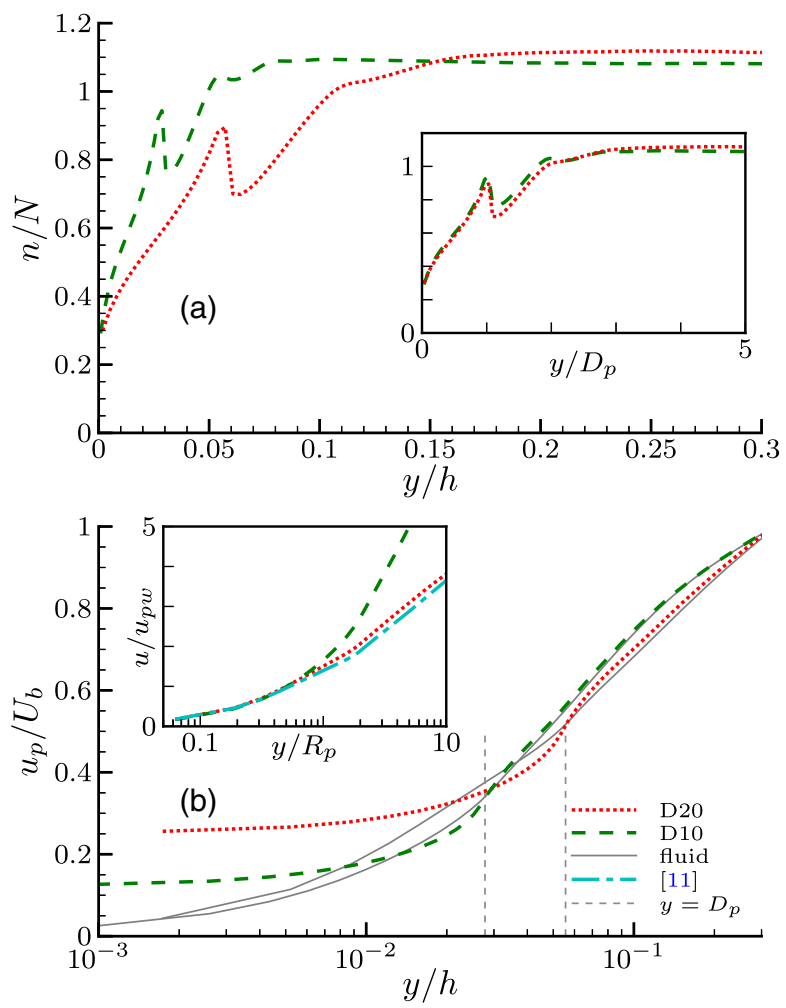

FIG. 2. (a) Mean particle number density $n$ divided by its bulk value $N$ versus $y / h$ in the main panel and $y / D_{p}$ in the inset (maximum statistical error within $95 \%$ confidence interval is $\pm 0.8 \%$ ). (b) Mean streamwise particle and fluid velocity. The inset shows the fluid velocity, normalized with $u_{p w}$ (definition in the text) versus $y / R_{p}$. The data from [11] pertain to the case of $\Phi=20 \%$. Vertical dashed lines depict a wall-normal distance of 1 particle diameter $\left(y=D_{p}\right)$ for cases D10 (closest to $\left.y=0\right)$ and D20; see Table I.

the length scale $\delta_{p w}$. The previous discussion showed that $\delta_{p w}$ scales with $D_{p}$ at fixed volume fraction. In addition, $\delta_{p w}$ should vanish in the single-phase limit, i.e. when $\Phi \rightarrow 0$. We therefore assume $\delta_{p w}$ (i) to be proportional to the solidity of the bulk suspension, measured as the ratio between particle size and mean particle separation distance, and (ii) to scale with the particle size. These hypotheses give the result above $\delta_{p w}=C\left(\Phi / \Phi_{\max }\right)^{1 / 3} D_{p}$, where the constant is set to $C=1.5$ [21] for all the cases addressed here and $\Phi_{\max }=0.6$. Note that displacing the origin of the turbulent region has been successfully adopted in turbulent flows over rough walls [22] but was not applied before to the case of turbulent suspensions.

In the same spirit, we further assume that the total stress $\tau=\rho u_{\tau}^{2}(1-y / h)$ acting across the channel is due to two distinct mechanisms. In the HSR, the increment in stress due to the particles is assumed to be well modeled by an effective suspension viscosity; in the particle-wall layer, instead, the stress increases due to the large apparent slip velocity near the wall. This is the main finite-size effect present in the flow. The stress in the HSR $\left(y>\delta_{p w}\right)$ corresponds therefore to that of a single-phase turbulent flow of a Newtonian fluid with viscosity $\nu^{e}$, in a channel with a wall origin at $y=\delta_{p w}$ and half-height $h-\delta_{p w}$. The flow in this region experiences an apparent stress $\rho u_{\tau}^{* 2} \leq \rho u_{\tau}^{2}$. In the particle-wall layer $\left(y<\delta_{p w}\right)$ the stress increases linearly when approaching the wall from $\rho u_{\tau}^{* 2}$ to $\rho u_{\tau}^{2}=\rho u_{\tau}^{* 2}+\Delta \tau_{p w}$. Hence, the total stress, linearly varying across the channel [11], is split into two contributions:

$$
\begin{aligned}
\tau= & \left(\rho u_{\tau}^{* 2}+\Delta \tau_{p w}\left(1-y / \delta_{p w}\right)\right) \mathcal{H}\left(\delta_{p w}-y\right) \\
& +\left(\rho u_{\tau}^{* 2}(h-y) /\left(h-\delta_{p w}\right)\right) \mathcal{H}\left(y-\delta_{p w}\right),
\end{aligned}
$$

where $\mathcal{H}$ is the Heaviside step function with the halfmaximum convention. Evaluating Eq. (1) at $y=\delta_{p w}$ yields the friction velocity in this region $u_{\tau}^{*}=u_{\tau}\left(1-\delta_{p w} / h\right)^{1 / 2}$. Given $u_{\tau}^{*}, \nu^{e}$, and $\delta_{p w}$ we obtain the following laws for the inner $\left(u / u_{\tau}^{*}=F\left[\left(y-\delta_{p w}\right) u_{\tau}^{*} / \nu^{e}\right]\right)$ and outer scaling $\left(\left(U_{c}-u\right) / u_{\tau}^{*}=G\left[\left(y-\delta_{p w}\right) /\left(h-\delta_{p w}\right)\right]\right)$ of the mean velocity in the overlap region of the HSR:

$$
\begin{gathered}
\frac{u}{u_{\tau}^{*}}=\frac{1}{\kappa} \ln \left(\frac{y-\delta_{p w}}{\delta_{v}^{e *}}\right)+B, \\
\frac{U_{c}-u}{u_{\tau}^{*}}=-\frac{1}{\kappa} \ln \left(\frac{y-\delta_{p w}}{h-\delta_{p w}}\right)+B_{d},
\end{gathered}
$$

with $u_{\tau}^{*}=u_{\tau}\left(1-\delta_{p w} / h\right)^{1 / 2}, \delta_{v}^{e *}=\nu^{e} / u_{\tau}^{*} ; \kappa, B$, and $B_{d}$ retain the values of single-phase flow; here $\nu^{e} / \nu=[1+$ $\left.(5 / 4) \Phi /\left(1-\Phi / \Phi_{\max }\right)\right]^{2}[3]$. Figure 3 reports the mean velocity profiles from the present simulations and the cases from [11]. The figure shows a collapse of the profiles in the logarithmic region, except for the case FP [11] with $\Phi=20 \%$ (see Table I). This is expected from our model, because it is the only case for which the friction Reynolds number based on the scaling parameters of the HSR $\operatorname{Re}_{\tau}^{h s r}=\left(h-\delta_{p w}\right) / \delta_{v}^{e *}<100$. This implies that there is

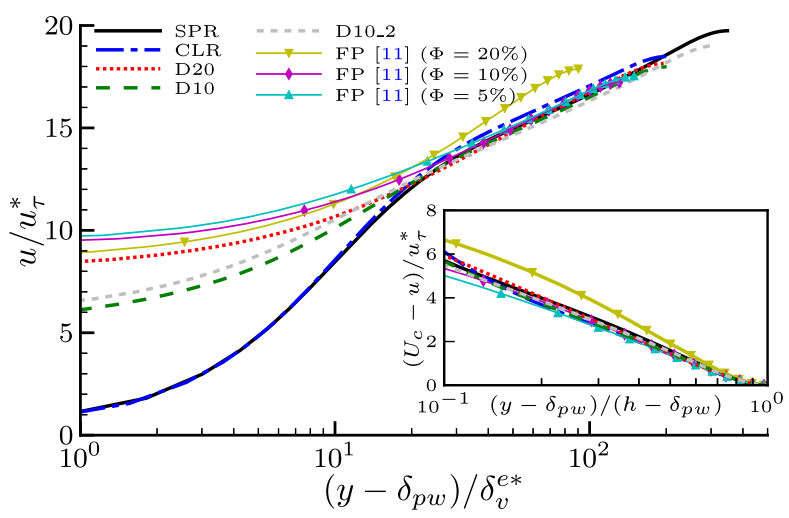

FIG. 3. Profiles of mean streamwise fluid velocity $u / u_{\tau}^{*}$ versus the wall-normal coordinate $\left(y-\delta_{p w}\right) / \delta_{v}^{e *}$. The inset shows the defect law, $\left(U_{c}-u\right) / u_{\tau}^{*}$, versus the distance to the wall in outer units $\left(y-\delta_{p w}\right) /\left(h-\delta_{p w}\right)$ (definitions in the text). Maximum statistical error is the same as in Fig. 1. 
not a sufficient separation of the inner and outer scales for the overlap region to exist [5], which is a necessary condition for the logarithmic scaling of the velocity profile. The defect law is shown in outer scaling in the inset of Fig. 3, where scaling in the logarithmic region can be clearly depicted. Also for this quantity the improvement with respect to the case where the particle-wall layer is not considered $\left(\delta_{p w}=0\right)$ is significant (not shown). Finally, the proposed scaling laws are used to derive the following drag law (i.e. the mean wall shear stress $\tau_{w}=\rho u_{\tau}^{2}$ ), expressed in terms of the friction Reynolds number:

$$
\operatorname{Re}_{\tau}=\frac{\operatorname{Re}_{b}}{2 \xi_{p w}^{1 / 2}}\left(\frac{1}{\kappa}\left[\ln \left(\operatorname{Re}_{\tau} \chi^{e} \xi_{p w}^{3 / 2}\right)-1\right]+B+B_{d}\right)^{-1},
$$

where $\xi_{p w}=\left(1-\delta_{p w} / h\right)$ and $\chi^{e}=\nu / \nu^{e}$. Equation (4) is derived in the same way as well-known laws from singlephase flow are derived [8]: by integrating the defect law [Eq. (3)] over the entire HSR to relate the bulk and centerline velocities, and combining Eqs. (2) and (3) to relate the friction and bulk velocities; see the Supplemental Material [23]. Note that Eq. (4) reduces to the well-known relation for single-phase flow when $\Phi \rightarrow 0$. Figure 4 compares the relative difference between the predicted values of $\mathrm{Re}_{\tau}$ and the values obtained from the DNS, $\operatorname{Re}_{\tau}^{d n s}$. The filled symbols correspond to predictions where only the effective viscosity is taken into account, i.e. $\delta_{p w}=0$, and the open symbols to predictions where both effects are accounted for. The estimates of the drag improve for the three data sets and the difference with the DNS values is less than $4 \%$. This supports the necessity of accounting for finite-size effects and further validates the proposed scaling. We remark that the implicit formulation of the drag law given by Eq. (4) can be replaced by a simple

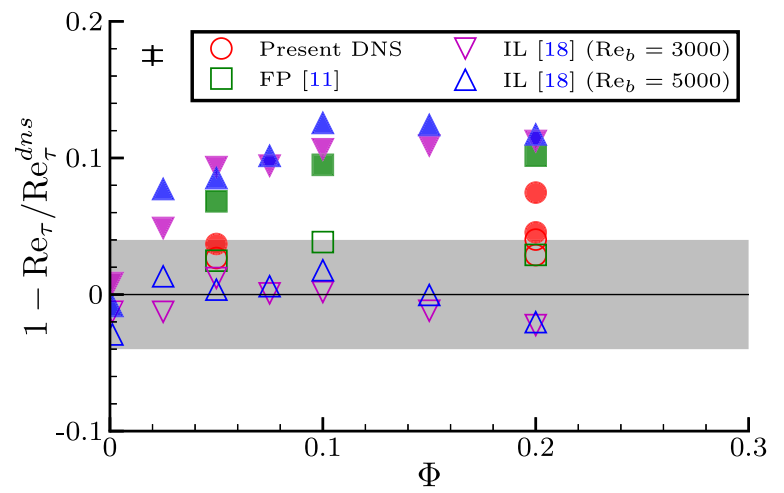

FIG. 4. Relative difference to the theoretical prediction of friction Reynolds number (the shaded area corresponds to a difference of $\pm 4 \%$ ). Filled symbols correspond to values that were not corrected for the presence of the particle-wall layer (i.e. $\delta_{p w}=0$ ). The maximum statistical error in the computation of the overall drag (with 95\% confidence interval) from the DNS is below $1 \%$. The corresponding (shifted) error bar is also shown on the left-hand side of the plot legend. explicit power law of $\operatorname{Re}_{\tau}$ as a function of $\operatorname{Re}_{b}$, less sensitive to insufficient inner-to-outer scale separation, which yields similar (and consistently, slightly more accurate at low Reynolds numbers) predictions for the drag; see Supplemental Material [23].

The solution of Eq. (4), normalized with the corresponding friction Reynolds number for single-phase flow $\operatorname{Re}_{\tau}^{s p h}=\left.\operatorname{Re}_{\tau}\right|_{\Phi=0}$, can be examined to draw general conclusions on the suspension behavior. For constant volume fraction and Reynolds number we conclude that a finite particle size causes a significant increase in drag with respect to the continuum limit due to the formation of a particle-wall layer. As expected, the drag increases monotonically with the particle size (corresponding to an increase of $\delta_{p w}$ ) and volume fraction (increasing $\delta_{p w}$ and $\nu^{e}$ ).

To conclude, we presented scaling laws for the mean velocity and the velocity defect in turbulent channel flow of neutrally buoyant finite-size spherical particles, which also enables us to accurately predict the total suspension drag. The model quantifies the main finite-size effect present in the flow-a particle-wall layer which always causes an increase in drag, by separating the dynamics of the flow in this layer and the homogeneous suspension region in the core. Exploiting conservation of momentum, this effect can be reduced to an apparent wall location $y=\delta_{p w}$ above which the flow is reasonably well represented by a Newtonian fluid with an effective suspension viscosity $\nu^{e}$. We validated our predictions for a reasonably wide range of the governing parameters.

This work was supported by the Portuguese Foundation for Science and Technology under Grant No. SFRH/BD/ $85501 / 2012$, by the European Research Council Grant No. ERC-2013-CoG-616186, TRITOS, by the Swedish Research Council (V. R.) and by COST Action MP1305: Flowing Matter. We acknowledge computer time provided by SNIC (Swedish National Infrastructure for Computing) and PRACE Project No. 2014112543 for awarding us access to resource CURIE based in France at Genci/CEA. Iman Lashgari is thanked for kindly providing the data from [18] in digital form.

*p.simoescosta@tudelft.nl

[1] S. Balachandar and J. K. Eaton, Annu. Rev. Fluid Mech. 42, 111 (2010).

[2] A. Einstein, Ann. Phys. (Berlin) 324, 289 (1906).

[3] J. J. Stickel and R. L. Powell, Annu. Rev. Fluid Mech. 37, 129 (2005).

[4] E. Guazzelli and J. F. Morris, A Physical Introduction to Suspension Dynamics, Vol. 45 (Cambridge University Press, Cambridge, 2011).

[5] S. B. Pope, Turbulent Flows (Cambridge University Press, Cambridge, 2000). 
[6] T. von Kármán, Nachrichten von der Gesellschaft der Wissenschaften zu Göttingen, Mathematisch-Physikalische 1930, 58 (1930).

[7] A. J. Smits, B. J. McKeon, and I. Marusic, Annu. Rev. Fluid Mech. 43, 353 (2011).

[8] R. Dean, J. Fluids Eng. 100, 215 (1978).

[9] V. C. Patel, W. Rodi, and G. Scheuerer, AIAA J. 23, 1308 (1985).

[10] J.-P. Matas, J. F. Morris, and E. Guazzelli, Phys. Rev. Lett. 90, 014501 (2003).

[11] F. Picano, W.-P. Breugem, and L. Brandt, J. Fluid Mech. 764, 463 (2015).

[12] A. Prosperetti, J. Fluid Mech. 768, 1 (2015).

[13] W.-P. Breugem, J. Comput. Phys. 231, 4469 (2012).

[14] R. A. Lambert, F. Picano, W.-P. Breugem, and L. Brandt, J. Fluid Mech. 733, 528 (2013).

[15] P. Costa, B. J. Boersma, J. Westerweel, and W.-P. Breugem, Phys. Rev. E 92, 053012 (2015).
[16] F. Picano, W.-P. Breugem, D. Mitra, and L. Brandt, Phys. Rev. Lett. 111, 098302 (2013).

[17] I. Lashgari, F. Picano, W.-P. Breugem, and L. Brandt, Int. J. Multiphase Flow 78, 12 (2016).

[18] I. Lashgari, F. Picano, W.-P. Breugem, and L. Brandt, Phys. Rev. Lett. 113, 254502 (2014).

[19] R. E. Hampton, A. A. Mammoli, A. L. Graham, N. Tetlow, and S. A. Altobelli, J. Rheol. 41, 621 (1997).

[20] K. Yeo and M. R. Maxey, J. Fluid Mech. 682, 491 (2011).

[21] Value obtained by fitting the agreement of the drag to the simulation results.

[22] P. S. Jackson, J. Fluid Mech. 111, 15 (1981).

[23] See Supplemental Material at http://link.aps.org/ supplemental/10.1103/PhysRevLett.117.134501 for the derivation of Eq. (4) and an alternative, explicit correlation for the overall drag. 